International Mathematical Forum, 1, 2006, no. 17, 797-808

\title{
Linear Estimation of Discrete Dynamic Systems with Incomplete Signals
}

\author{
C. Sánchez González, T.M. García Muñoz \\ Universidad de Granada (Spain) \\ Department of Quantitative Methods for Economics and Business \\ Campus de Cartuja \\ E-18071 Granada, Spain
}

\begin{abstract}
In this paper we focus our attention on the estimation of dynamic discrete systems in which the observed signal is not always related to all components in the state vector, but on the contrary, it can be considered incomplete, since we do not know if a particular observed signal contains information regarding all components or not. We develop a very simple framework to deal with these situations by introducing Bernoulli random variables in the observation equation. The algorithm derived can be considered as an extension of Nahi [8] algorithm for uncertain observations.
\end{abstract}

Mathematics Subject Classification: 62M20, 60G35

Keywords: Kalman filtering; Uncertain observations; Incomplete signal

\section{Introduction}

Kalman seminal paper in 1960 [3] states a new approach to estimation procedures by introducing a model describing the internal system behaviour known as the state space model. This new procedure is based on a recursive algorithm to get a filter minimizing the mean squared error (MSE) of the state vector. Further research on this topic (Jazwinski [2], Meditch [7], Kalman [4], Kowalski \& Szynal [5]) allowed to extend that method in a more general framework regarding the noise distribution in the model. Computational advantages of recursive estimators made them very useful in almost any other applied research field. Research papers on this topic before 1969 assumed that the estimated signal was always part of the available observation for its estimation. That year, Nahi [8] obtained a recursive least-squares estimation technique for the 
state variable in systems which observations, sometimes, did not contain the signal and therefore consisted only of noise. This type of systems is often called systems with uncertain observations. As it had previously happened with the initial paper by Kalman, Nahi results have been extended in [1] \& [9] by imposing less restrictive hypothesis on the noise structure.

At the moment, the estimation problem of signals in systems with uncertain observations is seen as important research topic in the communications area. More specifically Sánchez González \& García Muñoz [9] have shown its relevance in some economic problems related to correct the declared incomes in inquiry. Our empirical work revealed that in many practical situations data used to model real world phenomena through dynamic systems included some observations for which part of the signal to estimate was included but other was not. In those cases the model did not exactly matches a system with uncertain observations. For this reason we decided to improve our knowledge about such kind of systems and we know introduce a new algorithm to estimate the state of a discrete system in which observations can include some elements of the state vector not being ensured the presence of the resting others.

The rest of this article states as follows: section 2 is devoted to problem statement and to introduce the notation used. The third section includes the corresponding expressions for the filter and gain matrix algorithm and the corresponding variance-covariance matrices for filter and prediction errors. Proofs of the corresponding theorems are left to reader in Appendix. Finally, the fourth section includes some empirical evidence by comparison of results provided by this new proposed algorithm to Nahi and Kalman filters.

\section{Problem statement}

Let the following linear discrete-time dynamic system, with $n \times 1$ and $m \times 1$ state vectors $x_{1}(x)$ and $x_{2}(x)$, respectively, and $p \times 1$ observation vector $z(k)$, be given by

$$
\begin{aligned}
\left(\begin{array}{l}
x_{1}(k+1) \\
x_{2}(k+1)
\end{array}\right) & =\Phi(k+1, k)\left(\begin{array}{l}
x_{1}(k) \\
x_{2}(k)
\end{array}\right)+\Gamma(k+1, k) \omega(k), k \geq 0 \\
\left(\begin{array}{l}
x_{1}(0) \\
x_{2}(0)
\end{array}\right) & =\left(\begin{array}{l}
x_{10} \\
x_{20}
\end{array}\right) \\
z(k) & =H_{1}(k) x_{1}(k)+\gamma(k) H_{2}(k) x_{2}(k)+v(k), k \geq 0
\end{aligned}
$$

where $\Phi(k+1, k)=\left(\begin{array}{ll}\Phi_{11}(k+1, k) & \Phi_{12}(k+1, k) \\ \Phi_{21}(k+1, k) & \Phi_{22}(k+1, k)\end{array}\right), \Gamma(k+1, k), H_{1}(k)$ and $H_{2}(k)$ are known matrices of appropriate dimensions; $\{\omega(k), k \geq 0\}$ and $\{v(k), k \geq 0\}$ are white sequences with zero mean and $E\left[\omega(k) \omega^{T}(l)\right]=Q(k) \delta_{k l}$, 
$E\left[v(k) v^{T}(l)\right]=R(k) \delta_{k l}\left(\delta_{k l}\right.$ denotes the Kronecker function); $\left(\begin{array}{l}x_{10} \\ x_{20}\end{array}\right)$ is a random vector with zero mean and covariance matrix $\left(\begin{array}{cc}P_{11}(0) & P_{12}(0) \\ P_{12}^{T}(0) & P_{22}(0)\end{array}\right)$; $\{\gamma(k), k \geq 0\}$ is an independent sequence of Bernoulli random variables with $P[\gamma(k)=1]=p(k)$. It is assumed that $\left(\begin{array}{c}x_{10} \\ x_{20}\end{array}\right),\{\gamma(k), k \geq 0\},\{\omega(k), k \geq 0\}$ and $\{v(k), k \geq 0\}$ are independent each other.

As we can see the vector $x_{1}(k)$ is always included in the observation, but on the contrary, the presence of vector $x_{2}(k)$ can not be ensured. The probability of $x_{2}(k)$ not being included in the observation, $1-p(k)$, is sometimes known as false alarm probability.

Let $\widehat{x}_{1}(k / l)$ and $\widehat{x}_{2}(k / l)$ be the least mean squared error (LMSE) estimates of $x_{1}(k)$ and $x_{2}(k)$, respectively, given observations $z(0), \ldots, z(l)$. The estimation error is noted by

$$
e(k / l)=\left(\begin{array}{c}
e_{1}(k / l) \\
e_{2}(k / l)
\end{array}\right)=\left(\begin{array}{c}
x_{1}(k) \\
x_{2}(k)
\end{array}\right)-\left(\begin{array}{c}
\widehat{x}_{1}(k / l) \\
\widehat{x}_{2}(k / l)
\end{array}\right) .
$$

By

$$
P(k / l)=E\left[e(k / l) e^{T}(k / l)\right]=\left(\begin{array}{cc}
P_{11}(k / l) & P_{12}(k / l) \\
P_{21}^{T}(k / l) & P_{22}(k / l)
\end{array}\right)
$$

we mean the corresponding covariance matrices.

The LMSE linear filter and one-step ahead predictor of the state $\left(\begin{array}{l}x_{1}(k) \\ x_{2}(k)\end{array}\right)$ are presented in the next section.

\section{Prediction and filter algorithm}

Theorem 1. The predictor and filter of the state are given by

$$
\begin{aligned}
& \left(\begin{array}{l}
\widehat{x}_{1}(k+1 / k) \\
\widehat{x}_{2}(k+1 / k)
\end{array}\right)=\Phi(k+1, k)\left(\begin{array}{c}
\widehat{x}_{1}(k / k) \\
\widehat{x}_{2}(k / k)
\end{array}\right), k \geq 0 \\
& \left(\begin{array}{l}
\widehat{x}_{1}(0 /-1) \\
\widehat{x}_{2}(0 /-1)
\end{array}\right)=\left(\begin{array}{l}
0 \\
0
\end{array}\right) \\
& \left(\begin{array}{l}
\widehat{x}_{1}(k / k) \\
\widehat{x}_{2}(k / k)
\end{array}\right)=\left(\begin{array}{c}
\widehat{x}_{1}(k / k-1) \\
\widehat{x}_{2}(k / k-1)
\end{array}\right)+ \\
& F(k)\left[z(k)-\left(\begin{array}{ll}
H_{1}(k) & p(k) H_{2}(k)
\end{array}\right)\left(\begin{array}{l}
\widehat{x}_{1}(k / k) \\
\widehat{x}_{2}(k / k)
\end{array}\right)\right], \\
& k \geq 0
\end{aligned}
$$


where the filter gain matrix verifies

$$
F(k)=P(k / k-1)\left(\begin{array}{c}
H_{1}^{T}(k) \\
p(k) H_{2}^{T}(k)
\end{array}\right) \Pi^{-1}(k)
$$

and

$$
\begin{aligned}
\Pi(k)= & p(k)(1-p(k))\left(\begin{array}{ll}
0 & H_{2}(k)
\end{array}\right) S(k)\left(\begin{array}{c}
0 \\
H_{2}^{T}(k)
\end{array}\right)+ \\
& \left(\begin{array}{ll}
H_{1}(k) & \left.p(k) H_{2}(k)\right) P(k / k-1)
\end{array}\right)\left(\begin{array}{c}
H_{1}^{T}(k) \\
p(k) H_{2}^{T}(k)
\end{array}\right)+R(k), \quad k \geq 0
\end{aligned}
$$

with

$$
\begin{aligned}
S(k)= & \Phi(k, k-1) S(k-1) \Phi^{T}(k, k-1)+ \\
& \Gamma(k, k-1) Q(k-1) \Gamma^{T}(k, k-1), k>0 \\
S(0)= & \left(\begin{array}{ll}
P_{11}(0) & P_{12}(0) \\
P_{12}^{T}(0) & P_{22}(0)
\end{array}\right)
\end{aligned}
$$

The prediction and filter error covariance matrices satisfy

$$
\begin{aligned}
P(k+1 / k)= & \Phi(k+1, k) P(k / k) \Phi^{T}(k+1, k)+ \\
& \Gamma(k+1, k) Q(k) \Gamma^{T}(k+1, k), \quad k \geq 0 \\
P(0 /-1)= & \left(\begin{array}{cc}
P_{11}(0) & P_{12}(0) \\
P_{12}^{T}(0) & P_{22}(0)
\end{array}\right) \\
P(k / k)= & P(k / k-1)-F(k) \Pi(k) F^{T}(k), k \geq 0 .
\end{aligned}
$$

As we can observe, it is also possible to use this algorithm even in the case in which all elements of the state vector are present $\left(\Phi_{21}(k+1, k), \Phi_{22}(k+1, k)\right.$, $H_{2}(k)=0$ ), and degenerating in Kalman [3] algorithm, and also it can be used in case some of the elements in the state vector were not included in the observation $\left(\Phi_{11}(k+1, k), \Phi_{12}(k+1, k), H_{1}(k)=0\right)$, resulting in Nahi [8].

Note. The existence of the inverses of $\Pi(k)$, for $k \geq 0$, is not insured under the hypothesis of the system. If those don't exist, it will be used the corresponding pseudoinverses [6].

\section{Results}

In order to know more about the new algorithm behaviour, this is compared with Kalman and Nahi algorithms. "Mathematica" code has been performed for this task in order to generate signals and observations similar to those 
reported in this paper, including time-invariant system matrices and false alarm probability. Those observations have been used to get filters and signals through the three alternative algorithms: The one proposed in this paper along with Kalman's and Nahi's. Mean-squared errors (MSE) have been obtained to compare original simulated data and predicted values according these three algorithms.

Assuming $x_{1}(k)$ and $x_{2}(k)$ have scalar dimension $(1 \times 1)$, and different probability values to generate false alarms $(p=0.1,0.3,0.5,0.7,0.9)$, five simulations were obtained. System matrices were randomly generated. The following tables include the different results. The last three columns refer to the sums of MSE when comparing simulated real values for signals $x_{1}(k)$ and $x_{2}(k)$ to the corresponding predicted values through the three alternative algorithms.

\begin{tabular}{|c|c|c|c|c|}
\hline & Simulation & New MSE & Kalman MSE & Nahi MSE \\
\hline \multirow[t]{5}{*}{$1-p=0.9$} & 1 & $3.221 \times 10^{25}$ & $7.469 \times 10^{27}$ & $1.294 \times 10^{29}$ \\
\hline & 2 & $2.915 \times 10^{47}$ & $2.715 \times 10^{50}$ & $1.365 \times 10^{52}$ \\
\hline & 3 & 1.943 & 2.033 & 2.015 \\
\hline & 4 & $2.787 \times 10^{9}$ & $7.310 \times 10^{12}$ & $1.256 \times 10^{13}$ \\
\hline & 5 & 14.179 & 38.919 & 215.434 \\
\hline \multirow[t]{5}{*}{$1-p=0.7$} & 1 & 1.544 & 1.783 & 3.374 \\
\hline & 2 & 2.818 & 7.773 & 253.472 \\
\hline & 3 & 2884.49 & $5.320 \times 10^{6}$ & $4.132 \times 10^{7}$ \\
\hline & 4 & 160.332 & 8212.560 & 48.056 \\
\hline & 5 & 1.897 & 1.923 & 1.982 \\
\hline \multirow[t]{5}{*}{$1-0=0.5$} & 1 & 0.117 & 0.116 & 0.117 \\
\hline & 2 & 0.864 & 0.873 & 3.231 \\
\hline & 3 & 2.186 & 2.244 & 2.446 \\
\hline & 4 & $3.706 \times 10^{18}$ & $3.188 \times 10^{22}$ & $6.054 \times 10^{23}$ \\
\hline & 5 & 0.22495 & 0.22499 & 0.22526 \\
\hline \multirow[t]{5}{*}{$1-p=0.3$} & 1 & $2.235 \times 10^{45}$ & $2.156 \times 10^{47}$ & $2.566 \times 10^{47}$ \\
\hline & 2 & 2.210 & 2.227 & 2.158 \\
\hline & 3 & $1.013 \times 10^{31}$ & $3.613 \times 10^{32}$ & $4.453 \times 10^{33}$ \\
\hline & 4 & $3.388 \times 10^{41}$ & $1.046 \times 10^{43}$ & $3.228 \times 10^{44}$ \\
\hline & 5 & 6.148 & 6.277 & 6.211 \\
\hline \multirow[t]{5}{*}{$1-p=0.1$} & 1 & 1.472 & 1.471 & 1.455 \\
\hline & 2 & $7.477 \times 10^{18}$ & $6.357 \times 10^{20}$ & $8.128 \times 10^{19}$ \\
\hline & 3 & 673.668 & 1037.160 & 901.547 \\
\hline & 4 & 5.195 & 5.158 & 5.201 \\
\hline & 5 & 0.298 & 0.299 & 0.297 \\
\hline
\end{tabular}

As we can observe, when false alarm probability is high (for instance, $1-p=$ 0.9 ), the estimator supplied in this paper behaves relatively better in terms of lower MSE when compared to other two procedures by Kalman and Nahi. 
But, when false alarm probability decreases the three algorithms behave in a similar fashion although in most cases the new one takes advantage. In those cases in which the new procedure does not perform the best according to $\operatorname{MSE},(1-p=0.7$ [simulation 4 ], $1-p=0.5$ [simulation 1], $1-p=0.3$ [simulation 2], $1-p=0.1$ [simulations 1, 4, 5]), five additional simulations were performed but now using their particular system parameters for each case. Results obtained are included in the next table, and it can be easily confirm their behaviour is similar. 


\begin{tabular}{|c|c|c|c|c|}
\hline Parameters & Sim. & New MSE & Kalman MSE & Nahi MSE \\
\hline \multirow{5}{*}{$\begin{array}{l}1-p=0.7 \\
\Phi=\left(\begin{array}{ll}0.013 & 0.516 \\
0.656 & 0.697\end{array}\right) \\
\Gamma=\left(\begin{array}{l}0.040 \\
0.178\end{array}\right) \\
H_{1}=0.022 \\
H_{2}=0.185\end{array}$} & 1 & 21.4574 & 4125 & 85.412 \\
\hline & 2 & 809.054 & 41681 & 176.107 \\
\hline & 3 & 68.5789 & 10071 & 102.734 \\
\hline & 4 & 35.2527 & 275.262 & 98.3532 \\
\hline & 5 & 26.0756 & 82.3691 & 49.4407 \\
\hline \multirow{5}{*}{$\begin{array}{l}1-p=0.5 \\
\Phi=\left(\begin{array}{ll}0.078 & 0.541 \\
0.019 & 0.422\end{array}\right) \\
\Gamma=\left(\begin{array}{l}0.055 \\
0.146\end{array}\right) \\
H_{1}=0.048 \\
H_{2}=0.841\end{array}$} & 1 & 0.12632 & 0.127918 & 0.126297 \\
\hline & 2 & 0.114559 & 0.114925 & 0.114568 \\
\hline & 3 & 0.113073 & 0.113267 & 0.113092 \\
\hline & 4 & 0.116271 & 0.115148 & 0.116329 \\
\hline & 5 & $\begin{array}{c}0.0941957 \\
\end{array}$ & 0.0915039 & 0.0943239 \\
\hline \multirow{5}{*}{$\begin{array}{l}1-p=0.3 \\
\Phi=\left(\begin{array}{ll}0.455 & 0.501 \\
0.322 & 0.365\end{array}\right) \\
\Gamma=\left(\begin{array}{l}0.507 \\
0.284\end{array}\right) \\
H_{1}=0.182 \\
H_{2}=0.092\end{array}$} & 1 & 2.07364 & 2.06433 & 2.1188 \\
\hline & 2 & 4.00665 & 4.01239 & 4.01099 \\
\hline & 3 & 1.69426 & 1.71277 & 1.64679 \\
\hline & 4 & 2.42612 & 2.40028 & 2.53271 \\
\hline & 5 & 2.58135 & 2.58443 & 2.58818 \\
\hline \multirow{5}{*}{$\begin{array}{l}1-p=0.1 \\
\Phi=\left(\begin{array}{ll}0.147 & 0.071 \\
0.221 & 0.065\end{array}\right) \\
\Gamma=\left(\begin{array}{l}0.788 \\
0.620\end{array}\right) \\
H_{1}=0.684 \\
H_{2}=0.777\end{array}$} & 1 & 2.02868 & 2.02987 & 2.02852 \\
\hline & 2 & 1.63663 & 1.64155 & 1.6345 \\
\hline & 3 & 1.29962 & 1.30057 & 1.3001 \\
\hline & 4 & 1.69843 & 1.69317 & 1.70868 \\
\hline & 5 & 1.59178 & 1.59385 & 1.59257 \\
\hline \multirow{5}{*}{$\begin{array}{l}1-p=0.1 \\
\Phi=\left(\begin{array}{ll}0.560 & 0.088 \\
0.605 & 0.479\end{array}\right) \\
\Gamma=\left(\begin{array}{l}0.007 \\
0.547\end{array}\right) \\
H_{1}=0.690 \\
H_{2}=0.141\end{array}$} & 1 & 4.06456 & 4.12172 & 4.0577 \\
\hline & 2 & 4.98765 & 5.05695 & 4.9791 \\
\hline & 3 & 4.74853 & 4.76286 & 4.74707 \\
\hline & 4 & 3.34596 & 3.38284 & 3.34144 \\
\hline & 5 & 5.13663 & 5.13523 & 5.13724 \\
\hline \multirow{5}{*}{$\begin{array}{l}1-p=0.1 \\
\Phi=\left(\begin{array}{ll}0.060 & 0.667 \\
0.598 & 0.231\end{array}\right) \\
\Gamma=\left(\begin{array}{l}0.743 \\
0.230\end{array}\right) \\
H_{1}=0.047 \\
H_{2}=0.054\end{array}$} & 1 & 0.249804 & 0.250135 & 0.249395 \\
\hline & 2 & 0.301165 & 0.301718 & 0.300609 \\
\hline & 3 & 0.304371 & 0.305167 & 0.303564 \\
\hline & 4 & 0.312399 & 0.312504 & 0.312208 \\
\hline & 5 & 0.368456 & 0.368461 & 0.368756 \\
\hline
\end{tabular}




\section{Appendix A. Proof of Theorem1}

\section{(A.1) Predictor}

We see that $\Phi(k+1, k)\left(\begin{array}{l}\widehat{x}_{1}(k / k) \\ \widehat{x}_{2}(k / k)\end{array}\right)$ verifies the Orthogonal Projection Lemma $(\mathrm{OPL})$, that is to say,

$$
E\left[\left[\left(\begin{array}{c}
x_{1}(k) \\
x_{2}(k)
\end{array}\right)-\left(\begin{array}{c}
\widehat{x}_{1}(k / j) \\
\widehat{x}_{2}(k / j)
\end{array}\right)\right] z^{T}(\alpha)\right]=0, \alpha=0, \ldots, j
$$

By the state equation and since $\{\omega(k), k \geq 0\}$ is centered white noise independent from $\left(\begin{array}{l}x_{10} \\ x_{20}\end{array}\right),\{\gamma(k), k \geq 0\}$ y $\{v(k), k \geq 0\}$,

$$
E\left[\left(\begin{array}{l}
x_{1}(k+1) \\
x_{2}(k+1)
\end{array}\right) z^{T}(\alpha)\right]=\Phi(k+1, k) E\left[\left(\begin{array}{c}
x_{1}(k) \\
x_{2}(k)
\end{array}\right) z^{T}(\alpha)\right], \alpha=0, \ldots, k
$$

and by (1),

$E\left[\left(\begin{array}{c}x_{1}(k+1) \\ x_{2}(k+1)\end{array}\right) z^{T}(\alpha)\right]=\Phi(k+1, k) E\left[\left(\begin{array}{c}\widehat{x}_{1}(k / k) \\ \widehat{x}_{2}(k / k)\end{array}\right) z^{T}(\alpha)\right], \alpha=0, \ldots, k$.

Therefore, $\left(\begin{array}{c}\widehat{x}_{1}(k+1 / k) \\ \widehat{x}_{2}(k+1 / k)\end{array}\right)=\Phi(k+1, k)\left(\begin{array}{c}\widehat{x}_{1}(k / k) \\ \widehat{x}_{2}(k / k)\end{array}\right)$. In the initial instant, the estimate of $\left(\begin{array}{l}x_{1}(0) \\ x_{2}(0)\end{array}\right)$ is its mean, so that $\left(\begin{array}{l}\widehat{x}_{1}(0 /-1) \\ \widehat{x}_{2}(0 /-1)\end{array}\right)=0$.

\section{(A.2) Filter}

As a consequence of the orthogonal projection theorem, the state filter can be written as a function of the one-step ahead predictor as

$$
\left(\begin{array}{l}
\widehat{x}_{1}(k / k) \\
\widehat{x}_{2}(k / k)
\end{array}\right)=\left(\begin{array}{c}
\widehat{x}_{1}(k / k-1) \\
\widehat{x}_{2}(k / k-1)
\end{array}\right)+F(k) \delta(k), k \geq 0
$$

where $\delta(k)=z(k)-\widehat{z}(k / k-1)$ is the innovation process, and its expression is obtained below.

By the OPL, $\widehat{z}(k / k-1)$ is the only element of the subspace generated by the observations $\{z(0), \ldots, z(k-1)\}$ that verifies

$$
E\left[z(k) z^{T}(\alpha)\right]=E\left[\widehat{z}(k / k-1) z^{T}(\alpha)\right], \alpha=0, \ldots, k-1
$$

By the observation equation, given that the independent sequence $\{\gamma(k), k \geq 0\}$ is, in its turn, independent of $\left(x_{0},\{\omega(k), k \geq 0\},\{v(k), k \geq 0\}\right)$ and since $\{v(k), k \geq 0\}$ is independent white noise, we have

$$
E\left[z(k) z^{T}(\alpha)\right]=H_{1}(k) E\left[x_{1}(k) z^{T}(\alpha)\right]+p(k) H_{2}(k) E\left[x_{2}(k) z^{T}(\alpha)\right]
$$


and, by the OPL,

$$
\begin{gathered}
E\left[z(k) z^{T}(\alpha)\right]=E\left[\left\{H_{1}(k) \widehat{x}_{1}(k / k-1)+p(k) H_{2}(k) \widehat{x}_{2}(k / k-1)\right\} z^{T}(\alpha)\right], \\
\alpha=0, \ldots, k-1
\end{gathered}
$$

By (3), we conclude that $\widehat{z}(k / k-1)=H_{1}(k) \widehat{x}_{1}(k / k-1)+p(k) H_{2}(k) \widehat{x}(k / k-1)$, therefore, the innovation process is given by

$$
\delta(k)=z(k)-H_{1}(k) \widehat{x}_{1}(k / k-1)+p(k) H_{2}(k) \widehat{x}_{2}(k / k-1)
$$

and the filter expression is

$\widehat{x}(k / k)=\widehat{x}(k / k-1)+F(k)\left[z(k)-H_{1}(k) \widehat{x}_{1}(k / k-1)+p(k) H_{2}(k) \widehat{x}(k / k-1)\right]$.

\section{(A.3) Gain matrix.}

By (2), the filter error verifies $e(k / k)=e(k / k-1)-F(k) \delta(k)$. Since $e(k / k)$ is orthogonal to the observation $z(k)$, then $E\left[e(k / k) z^{T}(k)\right]=0$ and $E\left[e(k / k-1) z^{T}(k)\right]=F(k) E\left[\delta(k) z^{T}(k)\right]$. As $\widehat{z}(k / k-1)$ is orthogonal to $\delta(k)$, the covariance matrices of the innovation process verify $\Pi(k)=E\left[\delta(k) z^{T}(k)\right]$, then

$$
E\left[e(k / k-1) z^{T}(k)\right]=F(k) \Pi(k)
$$

and

$$
\left.F(k)=E\left[e(k / k-1) z^{T}(k)\right] \Pi^{-1}(k)=\left(\begin{array}{c}
E\left[e_{1}(k / k-1) z^{T}(k)\right. \\
E\left[e_{2}(k / k-1) z^{T}(k)\right.
\end{array}\right]\right) \Pi^{-1}(k) .
$$

By the observation equation, given that $\{\gamma(k), k \geq 0\}$, the initial state and $(\{\omega(k), k \geq 0\},\{v(k), k \geq 0\})$ are independent, and since $\{v(k), k \geq 0\}$ is centered white noise, we have

$$
\begin{aligned}
E\left[e_{1}(k / k-1) z^{T}(k)\right]= & E\left[e_{1}(k / k-1) x_{1}^{T}(k)\right] H_{1}^{T}(k)+ \\
& p(k) E\left[e_{1}(k / k-1) x_{2}^{T}(k)\right] H_{2}^{T}(k)
\end{aligned}
$$

and, since the estimation errors are orthogonal to the estimates,

$$
E\left[e_{1}(k / k-1) z^{T}(k)\right]=P_{11}(k / k-1) H_{1}^{T}(k)+p(k) P_{12}(k / k-1) H_{2}^{T}(k) .
$$

In the same fashion it can be shown that

$$
E\left[e_{2}(k / k-1) z^{T}(k)\right]=P_{12}^{T}(k / k-1) H_{1}^{T}(k)+p(k) P_{22}(k / k-1) H_{2}^{T}(k) .
$$


Then the expression of $F(k)$ is

$$
\begin{aligned}
F(k) & =\left(\begin{array}{c}
P_{11}(k / k-1) H_{1}^{T}(k)+p(k) P_{12}(k / k-1) H_{2}^{T}(k) \\
P_{12}^{T}(k / k-1) H_{1}^{T}(k)+p(k) P_{22}(k / k-1) H_{2}^{T}(k)
\end{array}\right) \Pi^{-1}(k) \\
& =P(k / k-1)\left(\begin{array}{c}
H_{1}^{T} \\
p(k) H_{2}^{T}
\end{array}\right) \Pi^{-1}(k) .
\end{aligned}
$$

(A.4) Covariance matrices of the innovation process, $\Pi(k)=E\left[\delta(k) \delta^{T}(k)\right]$.

Given that $\widehat{z}(k / k-1)$ is orthogonal to $\delta(k)$, we have $\Pi(k)=E\left[z(k) \delta^{T}(k)\right]$.

Substituting (4) in this equation

$$
\begin{aligned}
\Pi(k)= & E\left[z(k) z^{T}(k)\right]-H_{1}(k) E\left[\widehat{x}_{1}(k / k-1) z^{T}(k)\right]- \\
& p(k) H_{2}(k) E\left[\widehat{x}_{2}(k / k-1) z^{T}(k)\right] .
\end{aligned}
$$

By the properties imposed on the model,

$$
\begin{gathered}
E\left[z(k) z^{T}(k)\right]=H_{1}(k) E\left[x_{1}(k) x_{1}^{T}(k)\right] H_{1}^{T}(k)+ \\
p(k) H_{1}(k) E\left[x_{1}(k) x_{2}^{T}(k)\right] H_{2}^{T}(k)+ \\
p(k) H_{2}(k) E\left[x_{2}(k) x_{1}^{T}(k)\right] H_{1}^{T}(k)+ \\
p(k) H_{2}(k) E\left[x_{2}(k) x_{2}^{T}(k)\right] H_{2}^{T}(k)+R(k) \\
E\left[\widehat{x}_{1}(k / k-1) z^{T}(k)\right]=E\left[\widehat{x}_{1}(k / k-1) x_{1}^{T}(k)\right] H_{1}^{T}(k)+ \\
p(k) E\left[\widehat{x}_{1}(k / k-1) x_{2}^{T}(k)\right] H_{2}^{T}(k) \\
E\left[\widehat{x}_{2}(k / k-1) z^{T}(k)\right]=E\left[\widehat{x}_{2}(k / k-1) x_{1}^{T}(k)\right] H_{1}^{T}(k)+ \\
p(k) E\left[\widehat{x}_{2}(k / k-1) x_{2}^{T}(k)\right] H_{2}^{T}(k)
\end{gathered}
$$

Substituting all of these expressions in (6) and rearranging terms,

$$
\begin{aligned}
\Pi(k)= & H_{1}(k) E\left[e_{1}(k / k-1) x_{1}^{T}(k)\right] H_{1}^{T}(k)+ \\
& p(k) H_{1}(k) E\left[e_{1}(k / k-1) x_{2}^{T}(k)\right] H_{2}^{T}(k)+ \\
& p(k) H_{2}(k) E\left[e_{2}(k / k-1) x_{1}^{T}(k)\right] H_{1}^{T}(k)+ \\
& p(k) H_{2}(k) E\left[x_{2}(k) x_{2}^{T}(k)\right] H_{2}^{T}(k)+R(k)- \\
& p^{2}(k) H_{2}(k) E\left[\widehat{x}_{2}(k / k-1) x_{2}^{T}(k)\right] H_{2}^{T}(k) .
\end{aligned}
$$


If we denote by $S_{i j}=E\left[x_{i}(k) x_{j}^{T}(k)\right]$ and $S(k)=\left(\begin{array}{cc}S_{11}(k) & S_{12}(k) \\ S_{12}^{T}(k) & S_{22}(k)\end{array}\right)$,

$$
\begin{aligned}
\Pi(k)= & p(k)(1-p(k))\left(\begin{array}{ll}
0 & H_{2}(k)
\end{array}\right) S(k)\left(\begin{array}{c}
0 \\
H_{2}^{T}(k)
\end{array}\right)+ \\
& \left(\begin{array}{ll}
H_{1}(k) & \left.p(k) H_{2}(k)\right) P(k / k-1)
\end{array}\left(\begin{array}{c}
H_{1}^{T}(k) \\
p(k) H_{2}^{T}(k)
\end{array}\right)+R(k)\right.
\end{aligned}
$$

On the other hand, given that $\{\omega(k), k \geq 0\}$ is a white noise sequence and independent of $x_{0}$, the recursive expression of $S(k)$ is immediate.

\section{(A.5) Prediction and filter error covariance matrices.}

The expression of $P(k+1 / k)$ is immediate taking into account that the prediction error verifies:

$$
\begin{aligned}
e_{i}(k+1 / k)= & \Phi_{i 1}(k+1, k) e_{1}(k / k)+\Phi_{i 2}(k+1, k) e_{2}(k / k)+ \\
& \Gamma_{i}(k+1, k) \omega(k), \quad i=1,2 .
\end{aligned}
$$

In the other hand, for $i=1,2$, by the expression

$$
e_{i}(k / k)=e_{i}(k / k-1)-F_{i}(k) \delta(k),
$$

it is clear that

$$
\begin{aligned}
P_{i j}(k / k)= & P_{i j}(k / k-1)-E\left[e_{i}(k / k-1) \delta^{T}(k)\right] F_{j}^{T}(k)- \\
& F_{i}(k) E\left[\delta(k) e_{j}^{T}(k / k-1)\right]+F_{i}(k) \Pi(k) F_{j}^{T}(k)
\end{aligned}
$$

Since $\delta(k)=z(k)-H_{1}(k) \widehat{x}_{1}(k / k-1)+p(k) H_{2}(k) \widehat{x}_{2}(k / k-1)$, we have

$$
\begin{aligned}
E\left[e_{i}(k / k-1) \delta^{T}(k)\right]= & E\left[e_{i}(k / k-1) z^{T}(k)\right]- \\
& E\left[e_{i}(k / k-1) \widehat{x}_{1}^{T}(k / k-1)\right] H_{1}^{T}(k)- \\
& p(k) E\left[e_{i}(k / k-1) \widehat{x}_{2}^{T}(k / k-1)\right] H_{2}^{T}(k) .
\end{aligned}
$$

As the error is orthogonal to the estimates $\widehat{x}(k / k-1), i=1,2$, the two last terms are cancelled out and, taking into account (5),

$$
E\left[e_{i}(k / k-1) \delta^{T}(k)\right]=F_{i}(k) \Pi(k), i=1,2 .
$$

Substituting this expression in (7)

$$
P_{i j}(k / k)=P_{i j}(k / k-1)-F_{i}(k) \Pi(k) F_{j}^{T}, i=1,2
$$

and

$$
P(k / k)=P(k / k-1)-\left(\begin{array}{c}
F_{1}(k) \\
F_{2}(k)
\end{array}\right) \Pi(k)\left(\begin{array}{ll}
F_{1}^{T}(k) & \left.F_{2}^{T}(k)\right)
\end{array}\right.
$$




\section{References}

[1] A. Hermoso, J. Linares, Linear estimation for discrete-time systems in the presence of time-correlated disturbances and uncertain observations, IEEE Trans. Automatic Control, 39, No. 8 (1994), 1636-1638.

[2] A.H. Jazwinski, Stochastic Processes and Filtering Theory. Academic Press (1970).

[3] R.E. Kalman, A new approach to linear filtering and prediction problems, Trans. ASME Series D: Journal of basic engineering 82 (1960), 35-45.

[4] R.E. Kalman, New methods in Wiener filtering theory, Proc. Symp. Eng. Appl. Random Function Theory and Probability, 270-387, Wiley, New York (1963).

[5] A. Kowalski, D. Szynal, Filtering in discrete-time systems with state and observation noises correlated on a finite time interval, IEEE Trans. Automatic Control, AC-31, No.4 (1986), 381-384.

[6] J.R. Magnus, H. Neudecker, Matrix differential calculus with applications in Statistics an Econometrics, John Wiley and Sons (1988).

[7] J.S. Meditch, Stochastic Optimal Linear Estimation and Control. McGrawHill, New York (1969).

[8] N. Nahi, Optimal recursive estimation with uncertain observation, IEEE Trans. Inform. Theory IT-15 (1969), 457-462.

[9] C. Sánchez González, T.M. García Muñoz, Linear estimation for discrete systems with uncertain observations: an application to the correction of declared incomes in inquiry, Applied Mathematics and Computation 156 (2004), 211-233.

Received: September 28, 2005 